\title{
Elevated Liver Regeneration in Response to Pharmacological Reduction of Elevated Portal Venous Pressure by Terlipressin After Partial Hepatectomy
}

\author{
René Fahrner, ${ }^{1}$ Eleonora Patsenker, ${ }^{2}$ Andrea de Gottardi, ${ }^{1,2}$ Felix Stickel, ${ }^{2}$ Matteo Montani, ${ }^{3}$ \\ Deborah Stroka, ${ }^{1,2}$ Daniel Candinas, ${ }^{1,2}$ and Guido Beldi ${ }^{1,2,4}$
}

\begin{abstract}
Background. Liver regeneration is of crucial importance for patients undergoing living liver transplantations or extended liver resections and can be associated with elevated portal venous pressure, impaired hepatic regeneration, and postoperative morbidity. The aim of this study was to assess whether reduction of portal venous pressure by terlipressin improves postoperative liver regeneration in normal and steatotic livers after partial hepatectomy in a rodent model.

Methods. Portal venous pressure was assessed after minor (30\%), standard (60\%), or extended (80\%) partial hepatectomy $(\mathrm{PH})$ in mice with and without liver steatosis. Liver regeneration was assessed by BrdU incorporation and Ki-67 immunostaining.

Results. Portal venous pressure was significantly elevated post-PH in mice with normal and steatotic livers compared to sham-operated mice. Reduction of elevated portal pressure after $80 \% \mathrm{PH}$ by terlipressin was associated with an increase of hepatocellular proliferation. In steatotic livers, animals treated with terlipressin had an increase in liver regeneration after 30\% PH and increased survival after 60\% PH. Mechanistically, terlipressin alleviated IL-6 mRNA expression following PH and down-regulated p21 and GADD45 mRNA suggesting a reduction of cell cycle inhibition and cellular stress.
\end{abstract}

Conclusions. Reduction of elevated portal pressure post-PH by the use of terlipressin improves liver regeneration after PH in lean and steatotic mouse livers.

Keywords: Liver regeneration, Portal hypertension, Partial hepatectomy, Liver steatosis, Terlipressin.

(Transplantation 2014;97: 892-900)

O ptimal liver regeneration is of crucial relevance for the patient undergoing living liver donation and transplantation and liver resection. Removal of significant proportions of liver parenchyma is associated with an increase of portal blood flow and pressure (1-3). It has been shown that portal blood flow and pressure is increased immediately after partial hepatectomy $(\mathrm{PH})$ and triggers various signaling pathways $(4,5)$. However, the optimal range of portal blood flow and portal venous pressure required for best parenchymal regeneration after $\mathrm{PH}$ remains to be defined. Until now, the

This work has been supported by the Department of Clinical Research, University of Bern and the Foundation Durmüller Bol and the Swiss National Science Foundation.

The authors declare no conflicts of interest.

${ }^{1}$ Division of Visceral Surgery and Medicine, Inselspital, University Hospital Bern, Bern, Switzerland.

2 Department of Clinical Research, Inselspital, University Hospital Bern, Bern, Switzerland.

${ }^{3}$ Department of Pathology, Inselspital, University Hospital Bern, Bern, Switzerland.

${ }^{4}$ Address correspondence to: Guido Beldi, M.D., Division of Visceral Surgery and Medicine, University Hospital Bern, CH-3010 Bern, Switzerland.

E-mail: guido.beldi@insel.ch

R.F. participated in designing and performing the research, analyzing the data, and writing the article. E.P. participated in performing the research, analyzing the data, and writing the article. A.G. participated in designing the research, analyzing the data, and writing the article. F.S. only options that are clinically used to improve liver regeneration are invasive interventions such as preoperative embolization of portal vein branches $(6,7)$, splenic artery ligation $(8,9)$, splenectomy, or shunt operations $(2,10)$. However, despite their efficacy, these procedures are associated with additional surgical risks and potential morbidity.

The aim of this study was to explore whether pharmaceutical modulation of portal venous pressure in a non-cirrhotic liver optimizes liver regeneration and improves outcome after $\mathrm{PH}$. Terlipressin, a synthetic analog of vasopressin, effectively

participated in designing the research, analyzing the data, and writing the article. M.M. participated in designing and performing the research, analyzing the data, and writing the article. D.S. participated in designing the research, analyzing the data, and writing the article. D.C. participated in designing the research, analyzing the data, and writing the article. G.B. participated in designing the research, analyzing the data, and writing the article.

Supplemental digital content (SDC) is available for this article. Direct URL citations appear in the printed text, and links to the digital files are provided in the HTML text of this article on the journal's Web site (www.transplantjournal.com)

Received 28 May 2013. Revision requested 14 June 2013.

Accepted 30 December 2013.

Copyright (C) 2014 by Lippincott Williams \& Wilkins

ISSN: 0041-1337/14/9709-892

DOI: $10.1097 /$ TP.0000000000000045 

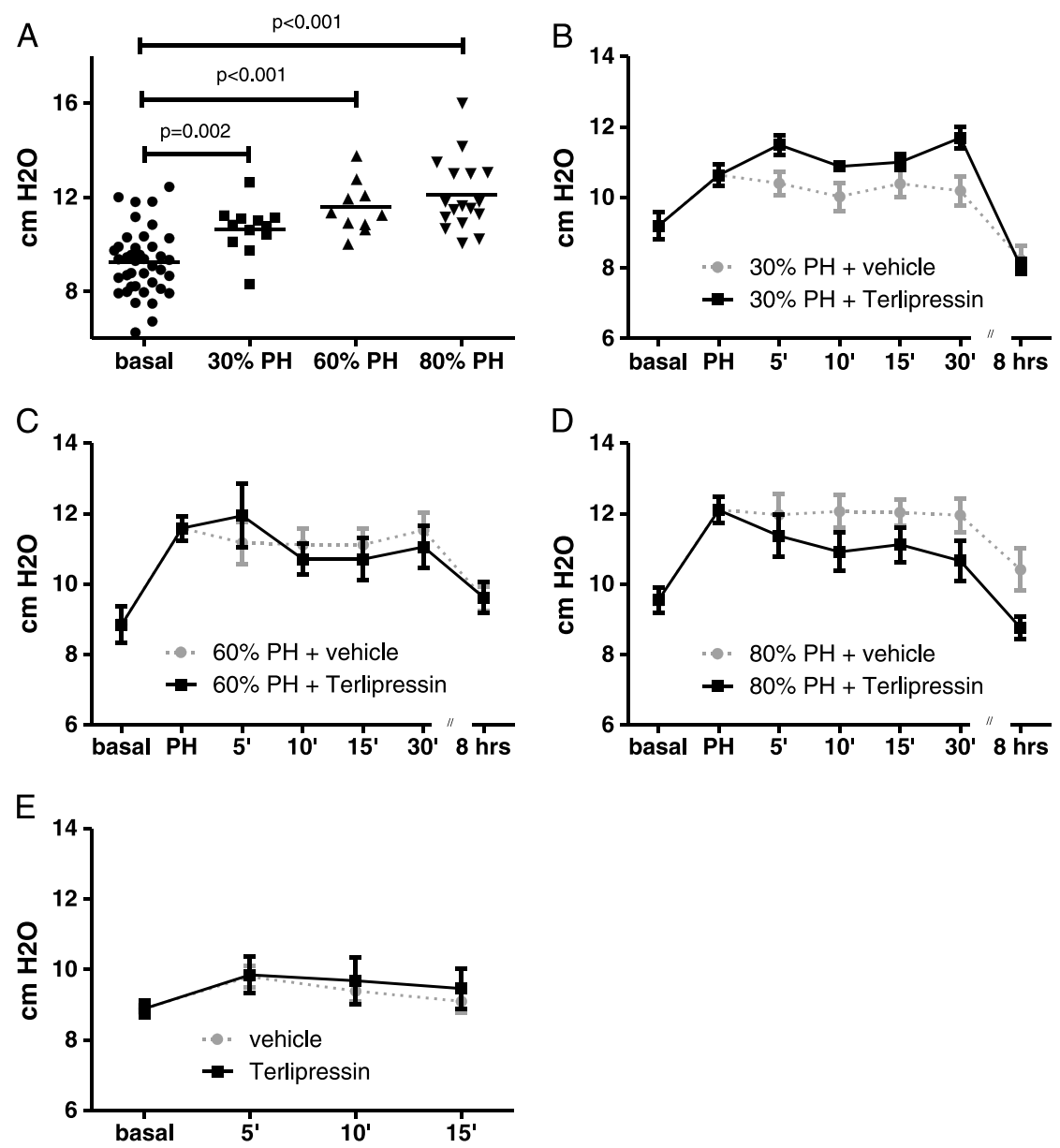

FIGURE 1. Invasive measurements of portal venous pressure before and after PH. A, portal venous pressure positively correlates with the extent of $\mathrm{PH}$ in mice after $30 \%, 60 \%$, and $80 \% \mathrm{PH}$ (Student $t$ test). B, after $30 \% \mathrm{PH}$, portal venous pressure remained significantly elevated in both groups compared to baseline with insignificant higher levels after terlipressin administration probably as a result of the mechanism of vasoconstriction of terlipressin $(P=0.2)$. C, portal venous pressure did not differ after $60 \% \mathrm{PH}$ between PBS- and terlipressin-treated animals $(P=0.5)$. D, after $80 \% \mathrm{PH}$, terlipressin administration was associated with significantly decreased portal venous pressure compared to controls over time $(P<0.001)$. E, after administration of terlipressin or PBS, portal venous pressure is not significantly different over time $(P=0.7)$. Animals $n=8$ per group and extent of $P H$. Mean, bars=SEM, two-way ANOVA test in (B) to (E).

reduces portal venous pressure in patients with liver cirrhosis and is currently recommended to lower portal venous pressure in the treatment of variceal bleeding and hepatorenal syndrome $(11,12)$.

Terlipressin acts as a vasoconstrictor via activation of vasopressin-1 receptors located in the smooth muscles of the arterial vasculature in the splanchnic region and thus decreases mesenteric and hepatic blood flow (13). A recent study proposed that terlipressin improves renal function and decreases portal venous pressure in patients undergoing donation for living-donor liver transplantation (14). However, no data on whether these circulatory effects impact liver parenchymal regeneration after $\mathrm{PH}$ are currently available.

Patients undergoing liver surgery with liver steatosis are an increasing problem in Western countries as liver steatosis is associated with an increased risk for postoperative complications $(15,16)$ and delayed liver regeneration in patients undergoing hepatic resection (17). In liver transplantation, elevated liver steatosis results in an increased graft failure (18).
Thus, hepatic steatosis that is associated with reduced functional capacity is of crucial clinical relevance. Therefore, the impact of terlipressin on portal venous pressure and liver regeneration post-PH was also evaluated in mice with and without steatosis.

The present study shows that terlipressin reduces portal venous pressure and improves outcome after extended $\mathrm{PH}$ in lean and steatotic livers. This effect is accompanied by improved liver cell regeneration mediated via the inhibition of proteins associated with cell cycle inhibition (p21) and cellular stress (GADD45) and modulation of interleukin (IL)-6 levels.

\section{RESULTS}

\section{Portal Venous Pressure is Modulated After PH in Response to Terlipressin}

In mice that underwent 30\% (minor), 60\% (standard), or $80 \%$ (extended) $\mathrm{PH}$, portal venous pressure positively correlated with the extent of liver resection with the highest pressure 
levels observed after 80\% PH (Fig. 1A). Terlipressin significantly decreased portal venous pressure in mice after $80 \%$, but not after $30 \%$ and $60 \% \mathrm{PH}$ compared to vehicle controls (Fig. 1B-D). Administration of terlipressin in sham-operated animals did not alter portal venous pressure (Fig. 1E).

\section{Liver Regeneration After $\mathbf{8 0} \% \mathbf{P H}$ is Increased in Response to Terlipressin}

Hepatocellular proliferation was used as a surrogate of parenchymal regeneration and was assessed by BrdU incorporation (Fig. 2A and B) and Ki-67 immunostaining (Fig. 2C and D)
A

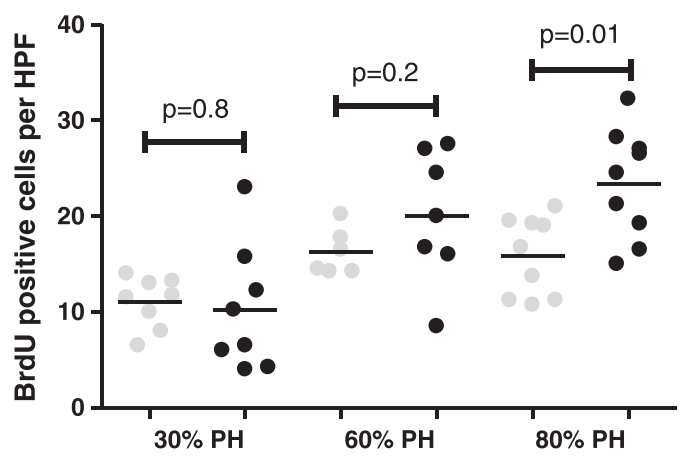

C

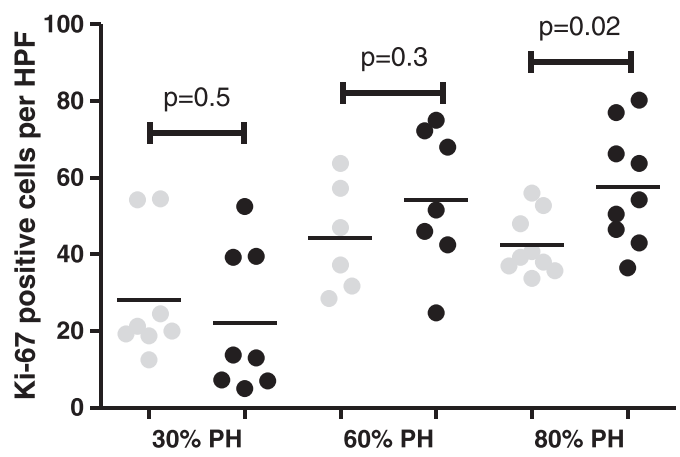

E

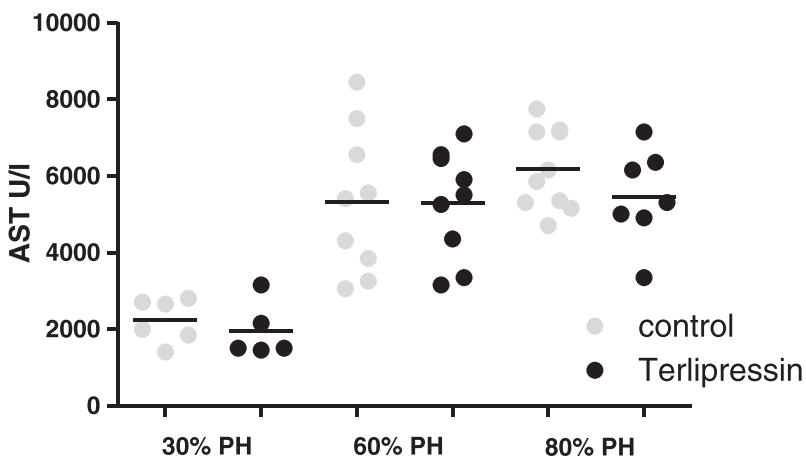

control

- Terlipressin

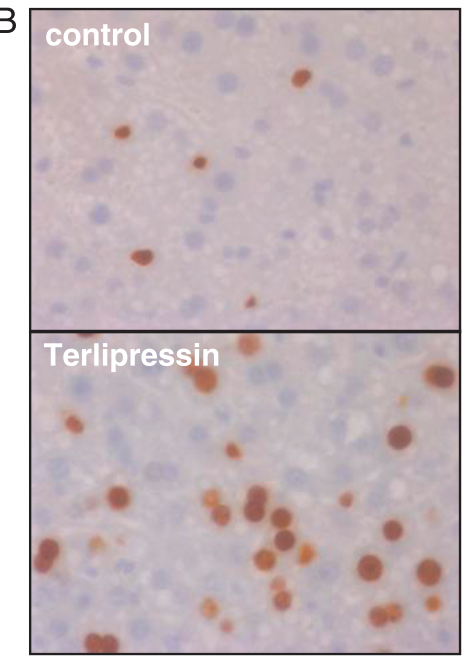

$\mathrm{D}$

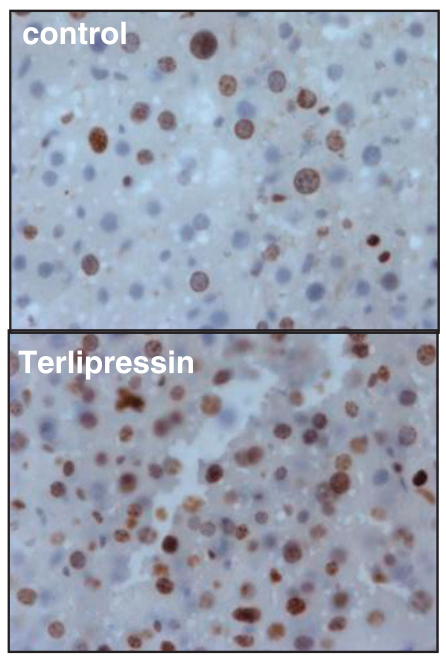

$\mathrm{F}$

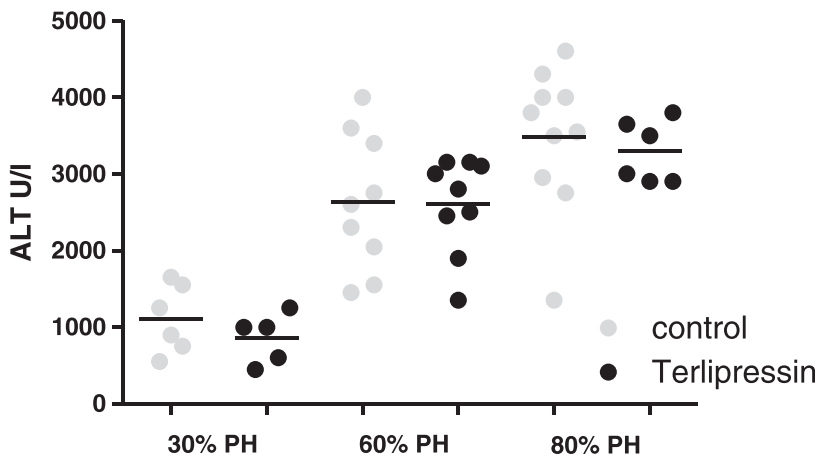

FIGURE 2. Immunohistochemical analysis with BrdU and Ki-67 staining after $48 \mathrm{hr}$ post-PH. A, C, significant increase of positive cells after terlipressin administration with $80 \% \mathrm{PH}$ compared to controls. B, D, representative liver sections of immunohistochemistry analysis of BrdU and Ki-67-positive hepatocytes. Assessment of liver injury by serum AST (E) and ALT (F) levels after $8 \mathrm{hr}$ post-PH. Horizontal lines indicate mean, Student $t$ test. 
A

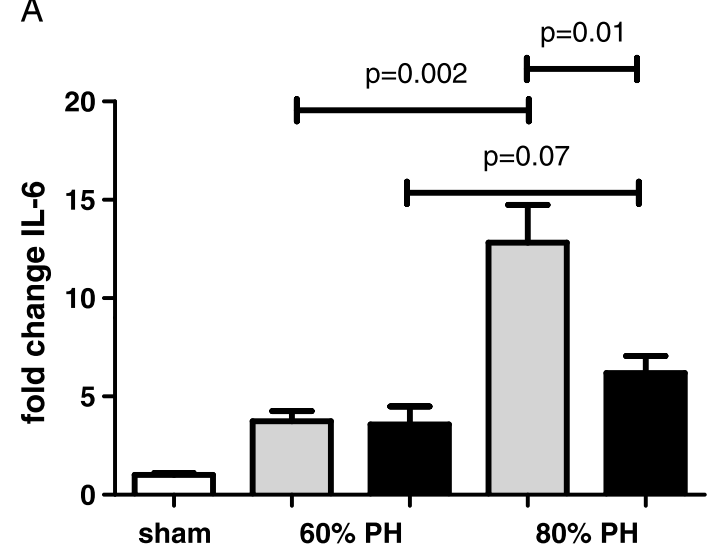

C

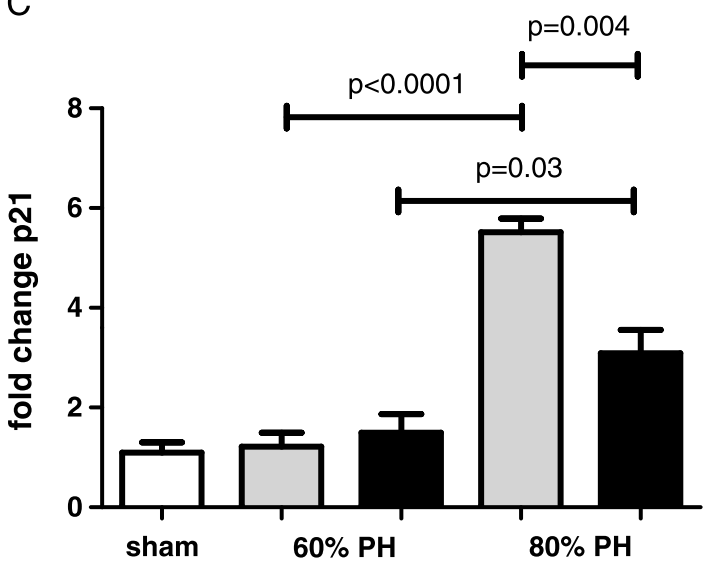

E

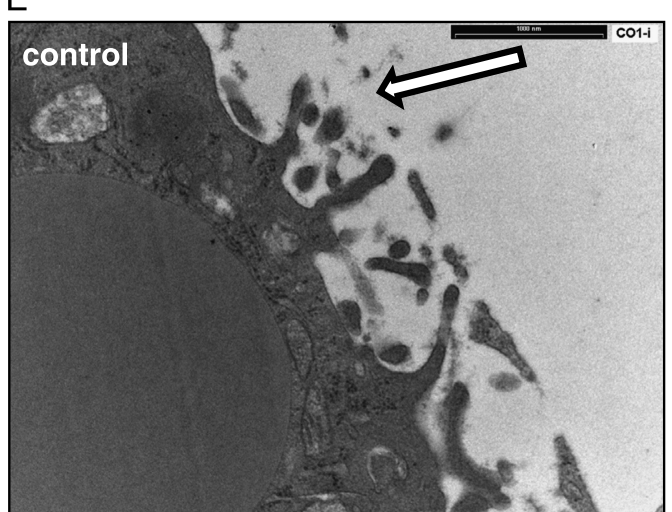

B

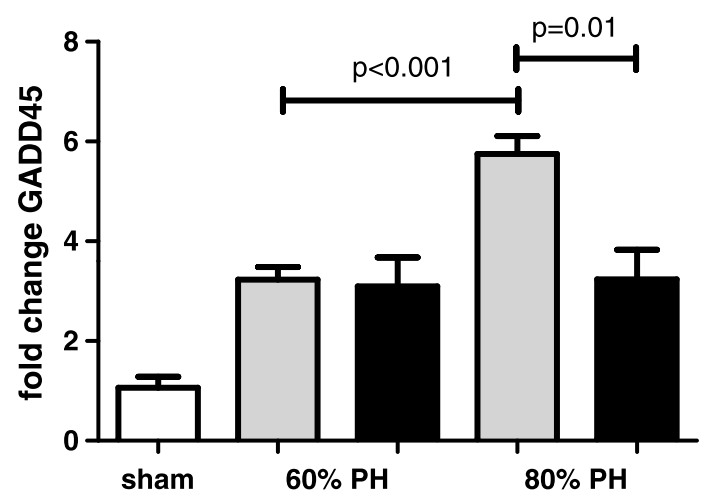

D

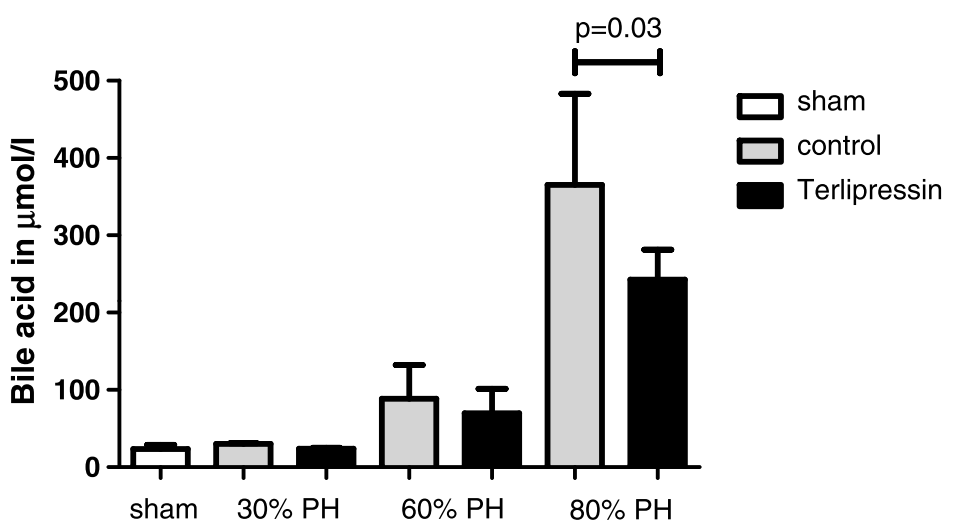

$\mathrm{F}$

FIGURE 3. Expression of hepatic mRNA of (A) IL-6 and (B) GADD45 $30 \mathrm{~min}$ post-PH and (C) p2l after $24 \mathrm{hr}$ assessed by quantitative RT-PCR were significantly increased after $80 \% \mathrm{PH}$ in comparison to $60 \% \mathrm{PH}$ in control animals. After terlipressin treatment, protein levels after $80 \% \mathrm{PH}$ decreased significantly, whereas after $60 \% \mathrm{PH}$ there were no significant changes. D, serum bile acid levels by ELISA after $48 \mathrm{hr}$ post-PH. Electron microscopy of liver sinusoids $4 \mathrm{hr}$ post-PH. After PBS administration and $80 \%$ PH (E), more disruption and destruction of sinusoids membrane was observed (arrow) compared to terlipressin administration (F). Mean, bars=SD, Student $t$ test.

$48 \mathrm{hr}$ after PH. Mice subjected to $30 \%$ and $60 \% \mathrm{PH}$ had substantial liver regenerative activity, which was not further increased by the administration of terlipressin. However, hepatocellular proliferation after $80 \% \mathrm{PH}$ was significantly enhanced in response to terlipressin. Serum transaminases (aspartate transaminase [AST] and alanine aminotransferase [ALT]) increased in accordance with the extent of $\mathrm{PH}$, reflecting hepatocyte injury, but there was no relevant difference between animals treated with terlipressin compared to vehicle controls
(Fig. 2E and F). Histology revealed no differences in terms of liver injury between the two treatment groups after $80 \% \mathrm{PH}$ (Fig. S1 A-F and Fig. S2 A-F, SDC, http://links.lww.com/TP/A948). An increase of microsteatotic changes and cell ballooning was seen with a maximum after $24 \mathrm{hr}$ after $\mathrm{PH}$ without changes after terlipressin administration. Histomorphology revealed foci of slight hepatocellular enlargement in lobular zone two. However, there were no differences between the mice treated with terlipressin and the control group. In addition, no elevation 
of hepatocellular proliferation was visible in sham-operated mice after terlipressin administration (data not shown).

\section{Modulation of Cytoline and Stress Responses}

The expression of proteins associated with a cytokine response (IL-6), cellular stress (GADD45), and cell cycle inhibition (p21) were examined post-PH with and without terlipressin. Expression of hepatic IL-6 mRNA was significantly lower postterlipressin administration compared to controls after $80 \% \mathrm{PH}$ but not after $60 \%$ (Fig. 3A). Proteins associated with cellular stress in response to partial hepatectomy, such as GADD45, and cell cycle inhibition, such as p21, were significantly decreased in animals treated with terlipressin compared to vehicle controls after $80 \%$ but not after $60 \% \mathrm{PH}$ (Fig. 3B and C). The significant differences occurred at $30 \mathrm{~min}$ post-PH for IL-6 and GADD45 and after 8 and 24 hr for p21 (Fig. S3 A-C, SDC, http://links.lww.com/TP/A948). An increase of serum bile acid levels as a marker of excretory biliary function post$\mathrm{PH}$ (19) was seen with the extent of PH in both treatment groups (Fig. 3D). Administration of terlipressin was associated with a significant decrease of bile acid levels in mice only after $80 \% \mathrm{PH}(P=0.03)$ but not in $30 \%$ or $60 \% \mathrm{PH}$. Electron microscopy revealed that the hepatic sinusoids were markedly
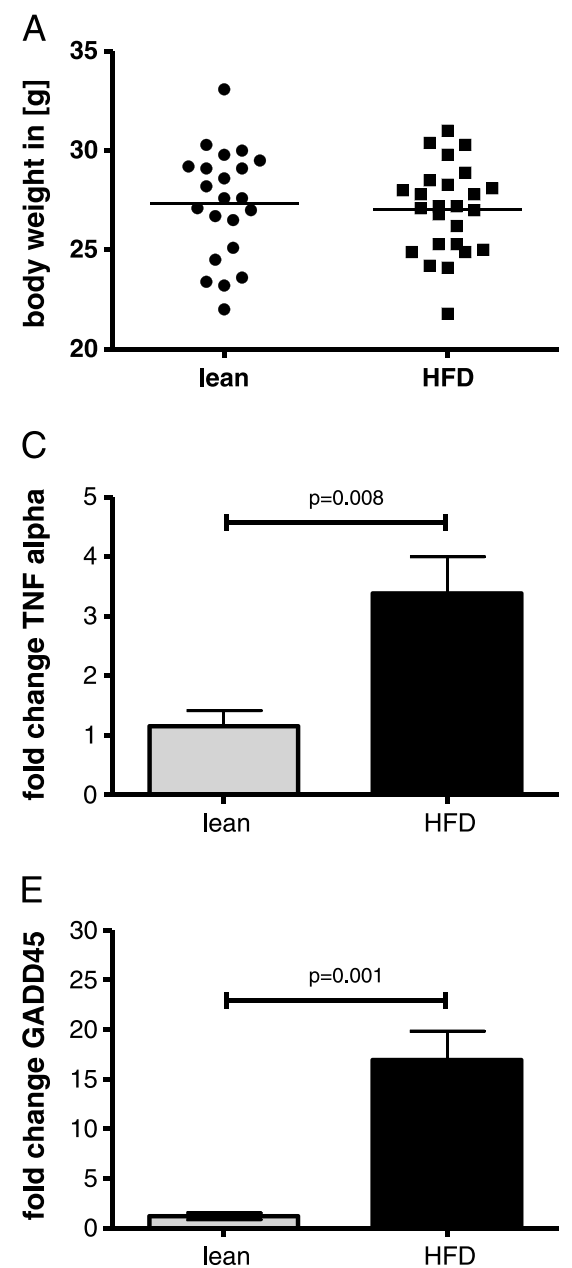

disrupted after $80 \% \mathrm{PH}$ (Fig. 3E). Staining for sinusoidal endothelial cells by CD31 revealed no significant differences between control and terlipressin-treated animals (Fig. S4 A-F, SDC, http://links.lww.com/TP/A948). However, by electron microscopy there was reduced disruption of endothelial cell damage in terlipressin-treated animals, suggesting that terlipressin protects the liver from microstructural sinusoidal damage caused by $80 \%$ PH (Fig. 3F).

\section{Portal Venous Pressure, Survival, and Liver Regeneration After PH in Liver Steatosis}

The effect of terlipressin on portal venous pressure, liver regeneration, and survival was explored in mice with steatotic livers. After 6 weeks on a high-fat diet (HFD), mice developed liver steatosis with a significant increase of liver weight but without changes in total body weight compared to control animals (Fig. 4A and B). Levels of tumor necrosis factor (TNF)- $\alpha, \mathrm{p} 21$, and GADD45 mRNA were significantly increased in mice with HFD compared to mice receiving standard chow indicating lipotoxicity from fat deposits in the liver, whereas IL-6 transcripts were significantly lower in mice with steatosis compared to mice with normal liver parenchyma (Fig. 4C-F). Steatotic changes and accumulation of intracellular fat droplets
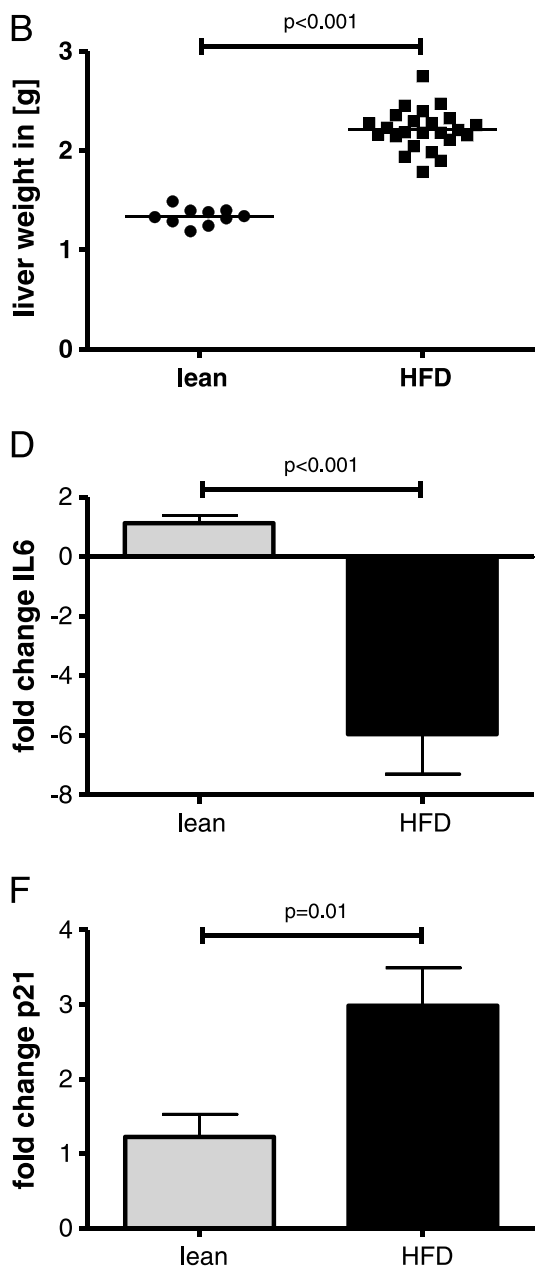

FIGURE 4. Comparison of mice fed a high fat diet (HFD) versus control diet. A, HFD for 6 weeks showed no effect on whole body weight. B, HFD was associated with a significant increase of liver weight. Expression of mRNA of (C) TNF alpha, (D) IL-6, (E) GADD45, and (F) p2 1 in liver tissues of lean and steatotic livers (HFD). Mean, bars=SD, Student $t$ test. 
A

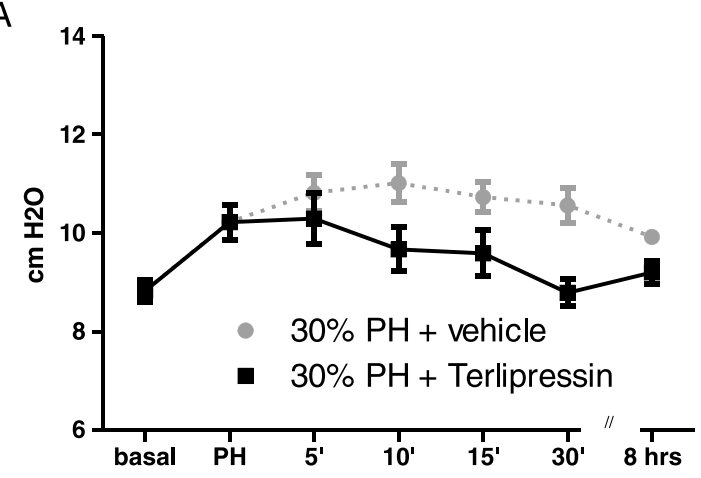

C

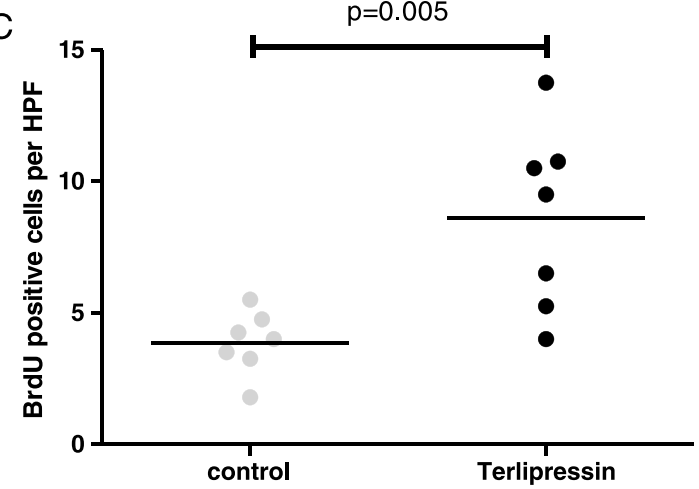

E

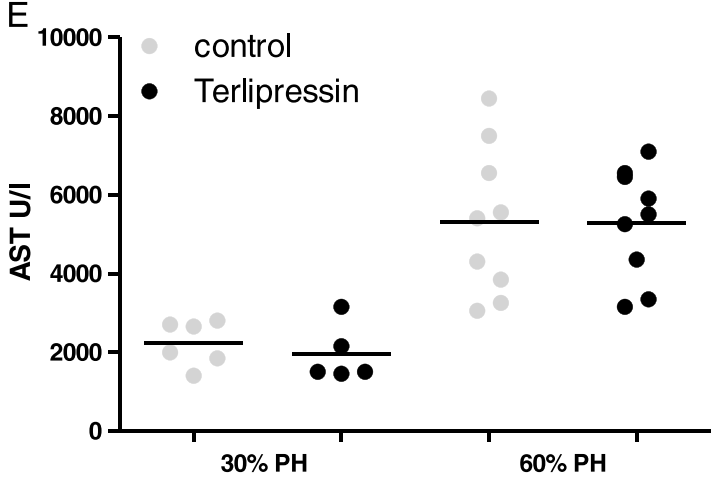

G

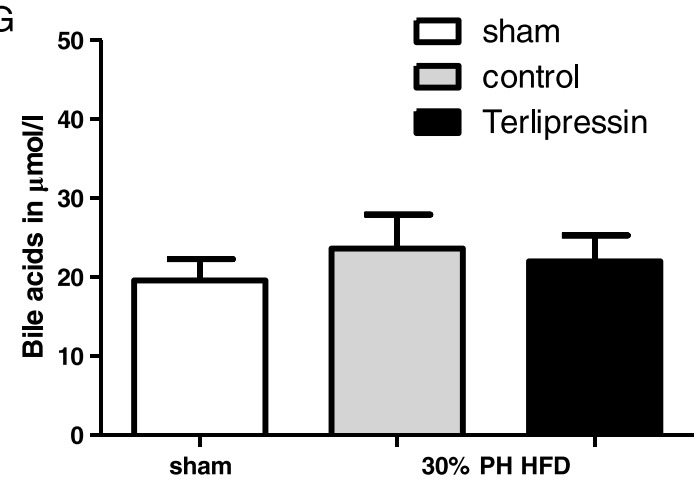

B

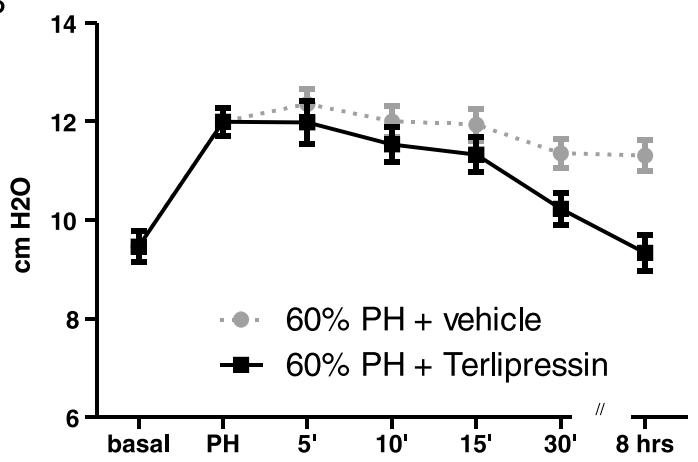

D

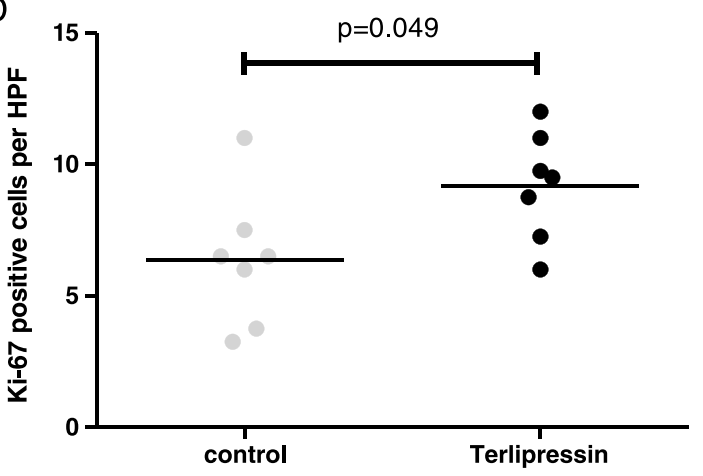

F

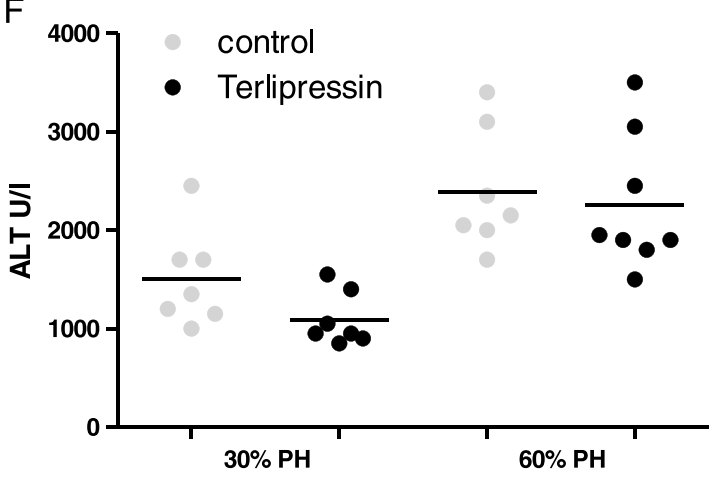

$\mathrm{H}$

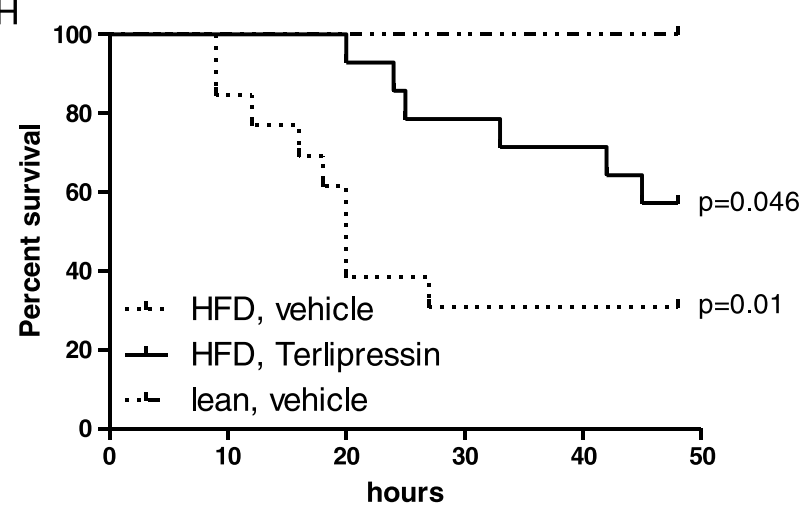

FIGURE 5. Effect of terlipressin treatment on portal venous pressure, liver regeneration, and liver injury after PH in animals treated with high-fat diet (HFD). A, B, portal venous pressure after $30 \%$ and $60 \%$ PH. Mean, bars=SEM, two-way ANOVA test. C, D, assessment of proliferation by BrdU and Ki-67 immunostaining after $48 \mathrm{hr}$ after 30\% PH. E, F, no effect of terlipressin on liver injury assessed by AST and ALT levels $8 \mathrm{hr}$ after 30\% PH. G, serum bile acid levels measured by ELISA after $48 \mathrm{hr}$ after $30 \% \mathrm{PH}$. Mean, bars=SD, Student $t$ test. H, significant improvement of survival after treatment with terlipressin after $60 \%$ PH. Kaplan-Meier survival curve, log rank. 
were not associated with an increase of basal portal venous pressure or hydroxyproline content of the liver, thereby excluding significant hepatic fibrosis in this model (Fig. S5 A-D, SDC, http://links.lww.com/TP/A948). These findings were confirmed by liver histology and revealed slight changes as hepatocellular degradation, cell ballooning, and mild inflammatory infiltrates, but no significant fibrosis (Fig. S5 E and F, SDC, http://links.lww.com/TP/A948). Administration of terlipressin in mice with liver steatosis was associated with decreased portal venous pressure after $30 \% \mathrm{PH}$ (Fig. 5A) and 60\% PH (Fig. 5B) compared to vehicletreated controls. Terlipressin significantly increased hepatic proliferation $48 \mathrm{hr}$ after 30\% PH (Fig. 5C and D), whereas it had no effect on liver injury as assessed by AST and ALT levels in 30\% and 60\% PH (Fig. 5E and F). Additionally, there were no significant changes in bile acid levels after $48 \mathrm{hr}$ after $30 \% \mathrm{PH}$ and HFD (Fig. 5G). For survival analysis, all animals were included that were enrolled for the 48-hr proliferation measurements. In mice with liver steatosis, survival was significantly lower after $60 \% \mathrm{PH}$ compared to control mice without HFD $(P=0.01)$, and administration of terlipressin was associated with a significantly improved survival ( $58 \%$ vs. $30 \%$ after $50 \mathrm{hr} ; P=0.046$; Fig. $5 \mathrm{H}$ ).

\section{DISCUSSION}

The data presented in this study provide evidence that elevated portal venous pressure following $\mathrm{PH}$ can be reduced by a pharmacological approach and improves parenchymal regeneration in mice. Importantly, terlipressin improved hepatocellular proliferation in mice after $80 \%$ hepatectomy and increased survival in mice with liver steatosis indicating a therapeutic potential for terlipressin in situations with a high risk of postoperative liver insufficiency. Mechanistically, hepatoprotection from terlipressin may be explained by reduced cellular stress as reflected by decreased levels of p 21 and GADD45 and altered cytokine response. Interestingly, terlipressin modulated regeneration but had no effect on liver injury.

An increase in portal venous pressure correlated with the extent of $\mathrm{PH}$ and was detected immediately following resection of liver lobes. Minor elevations of portal venous pressure after $\mathrm{PH}$ have been shown to be pivotal for the initiation of liver regeneration (20). Conversely, extended liver resections or small-for-size liver transplantations that are associated with a marked increase of portal venous pressure, beyond yet undefined levels, may impair the regenerative capacity of the liver and lead to liver failure (21). To be clinically applicable and to avoid side effects in animals with normal portal venous pressure, the administration of the drug was performed immediately after $\mathrm{PH}$. Therefore, the very first elevation of portal venous pressure was not altered. Despite this delayed injection, a positive effect on liver regeneration and reduced cellular stress after $80 \% \mathrm{PH}$ has been observed. The reduction of portal venous pressure at $8 \mathrm{hr}$ post-PH reflects early changes of hepatic remodeling and adaptation of the sinusoids to the alterations of pressure and flow in response to PH (22). Thus, maintaining optimal levels of postoperative portal venous pressure seems to be crucial for successful outcomes.

Administration of terlipressin was only protective after $80 \%$ PH. Thus, nonsignificant changes in portal venous pressure after $60 \% \mathrm{PH}$ after treatment with terlipressin are not sufficient to impact on liver regeneration. This finding is supported by increasing evidence that not the size alone but the flow in resected or transplanted livers play an important role in liver regeneration and "small for flow" rather than "small for size" seems to be critical (23).

Our report now shows for the first time that modulation of portal venous pressure by terlipressin is beneficial in the absence of liver cirrhosis. Up to now, vasopressin agonists have been shown to be clinically effective in lowering portal venous pressure in cirrhotic livers and terlipressin has been shown to improve short-term survival of patients with hepatorenal syndrome (24). Interestingly, a reduction of portal venous pressure by terlipressin occurs only after $80 \%$ $\mathrm{PH}$ that critically impact not just on portal venous pressure but also on splanchnic blood flow and systemic metabolism (25). The impact of physiological release of vasopressin on liver regeneration via $\mathrm{Ca}^{2+}$ signaling has been previously described $(5,26)$. These reports have shown neuroendocrine secretion of vasopressin from the hypothalamus in response to $\mathrm{PH}$ that protects the liver. We now support these mechanistic findings at the target organ and show that pharmacological activation of vasopressin receptors reduces portal venous pressure and is associated with an increase in liver regeneration.

There is little evidence whether terlipressin has direct stimulative potential on hepatocyte proliferation. No effect of terlipressin on hepatocellular proliferation or on portal venous pressure has been observed after minor or standard $\mathrm{PH}$. Thus, the main pathway of inducing hepatocyte proliferation is probably by means of lowering the portal venous pressure and consecutive reduced cellular stress rather than via direct hepatocyte V1a vasopressin receptors.

Terlipressin mainly acts through a vasoconstriction in the splanchnic vessels and therefore reduces the portal flow and portal venous pressure in cirrhosis and consecutive splanchnic vasodilatation. Hypothetically, terlipressin also counteracts a potential hepatic buffer response that may occur post-PH. The doses of terlipressin used in this study were comparable to previous reports in cirrhotic mice with effective reduction of portal pressure mainly after $80 \% \mathrm{PH}$ and without negative side effects on, for example, intestinal perfusion. In patients undergoing living liver transplantation, terlipressin administration was associated with an effective reduction of portal venous pressure and improvement of renal function without having negative systemic side effects (14).

Hepatic steatosis is a frequent parenchymal liver disease in Western countries. Liver transplantation of livers with modest steatosis is a potential clinical scenario, and it has been shown clinically and experimentally that it is associated with an increased risk for complications $(15,27)$. There was no mortality after $80 \% \mathrm{PH}$, but after $60 \% \mathrm{PH}$ in steatotic mice. Thus, a blunted response to this critical extent of PH may explain the effect of terlipressin at $60 \% \mathrm{PH}$ in steatotic mice.

In addition to the hemodynamic changes in response to terlipressin administration, we demonstrated reduced cellular stress (GADD45) and cell cycle inhibition (p21) after $80 \% \mathrm{PH}$. Both parameters were already significantly upregulated in steatotic livers before surgery, which explains the effect already at $60 \% \mathrm{PH}$. These results confirm recent studies that demonstrated a critical impact of both p21 and 
GADD45 on the initiation and the kinetics of liver regeneration $(28-30)$.

Our study also revealed that terlipressin reduced disruptions of hepatic sinusoids, which were associated with extended $\mathrm{PH}$. As previously shown in a transgenic animal model, portal hypertension is associated with a morphological change of sinusoidal fenestrations, and PH is characterized by a disappearance of the sieve-plate arrangement, endothelial fenestration, and dilatation of bile canaliculi $(31,32)$. The sinusoidal disruption as observed in the present study is potentially associated with an increased intrahepatic blood shunting between portal venous and systemic circulation. Therefore, metabolites of the liver such as bile acids might be found in higher concentrations in the systemic blood circulation because of increased blood shunting after extended $\mathrm{PH}$. It remains unclear if coincidence of reduced bile acids and reduced sinusoidal disruption in response to terlipressin administration is the consequence or the cause of improved outcome for the liver regeneration.

In summary, our results show pharmacological reduction of portal venous pressure with terlipressin leads to an improvement of liver regeneration, maintenance of microstructural hepatic tissue anatomy, a reduction of stress response genes, and, most importantly, a better survival.

\section{MATERIALS AND METHODS}

All procedures were carried out in accordance with the Swiss National Institutes of Health guidelines for the care and use of experimental animals, and the experimental protocol received approval by the Animal Care Committee of the Canton of Bern, Switzerland.

\section{Animal Preparation and Experimental Setting}

Experiments were performed on 8- to 12-week-old adult wild-type C57BL/6J mice obtained from Harlan Animal Research Laboratories (Boxmeer, The Netherlands). The animals were housed in the University Animal Facility with a $12 \mathrm{hr}$ light/dark cycle at $22^{\circ} \mathrm{C}$, and fed either with normal chow (fat: $4.5 \%$, protein: $18.5 \%$, fibers: $4.5 \%$ ) or with a high-fat diet (Ssniff, Soest, Germany) for 6 weeks (HFD—fat: 16.6\% [50\% lard and 50\% cacao butter], protein: $15.7 \%$, fibers: $4.5 \%$ ). Surgical procedures were performed under general anesthesia using isoflurane (Nicholas Piramal (I) Limited, London, UK). During the procedure, the intestine was rinsed with saline to avoid drying-out and resuscitation with saline of the intraoperative fluid loss was given at the end of the operation. Postoperative analgesia with buprenorphin (Reckitt Benckiser AG, Switzerland) was regularly administered subcutaneously during the postoperative course. At the time of sacrifice, mice were anesthetized with isoflurane inhalation, lethal blood samples were taken from the inferior vena cava, and livers were collected for further analyses.

\section{Partial Hepatectomy Model and Treatment With Terlipressin}

The details of the PH model in mice were previously published (33). In brief, resection of the median and left lobe equals a standard $60 \% \mathrm{PH}$, whereas for minor $\mathrm{PH}(30 \%)$ only the left superior lobe was excised. For extended $\mathrm{PH}(80 \%)$, the right inferior lobe was resected in addition to the standard $60 \%$ PH. Directly after performing $\mathrm{PH}$ and before closure of the abdomen, the animals received terlipressin $(0.05 \mu \mathrm{g} / \mathrm{g}$ mouse, Glypressin; Ferring Pharmaceuticals, Switzerland) or the vehicle phosphate-buffered saline (PBS; Gibco, Invitrogen, Auckland, New Zealand) as control $(5 \mu \mathrm{L} / \mathrm{g}$ mouse) intravenously injected into the inferior vena cava. After $8 \mathrm{hr}$, this administration was repeated by injections into the femoral vein under general anesthesia with isoflurane. Two hours before sacrifice, $50 \mathrm{mg} / \mathrm{kg}$ mouse of bromodeoxyuridine (BrdU, \#16880; Fluka, Sigma Aldrich, Switzerland) was injected intraperitoneally. For each extent of PH with or without HFD, there was a treatment and control group of each 8 to 12 animals per group and time point.

\section{Measurement of Portal Venous Pressure}

Portal venous pressure measurements were terminal experiments and were not used for survival analysis. After laparotomy the ileocecal vein was identified and a $26 \mathrm{G}$ catheter was inserted and the catheter tip placed close to the portal confluence and finally fixed with histoacryl. Baseline portal venous pressure was measured by a computer-based program (FlowChart 7.0; AD Instruments, Spechbach, Germany) during $1 \mathrm{~min}$. Portal venous pressure was measured at specific time points before and after $(5,10,15,30 \mathrm{~min}$ and $8 \mathrm{hr}) \mathrm{PH}$ and always required anesthesia and laparotomy.

\section{Histological and Immunohistochemical Analysis of the Liver}

Fresh liver tissue was fixed overnight in $4 \%$ paraformaldehyde in PBS and embedded in paraffin. Liver sections $(5 \mu \mathrm{m})$ were deparaffinized with xylol and counterstained with hematoxylin-eosin and with reticulin stain (Ag) for histological assessment. For immunohistochemistry, paraffin-embedded tissue sections were dried for $24 \mathrm{hr}$, deparaffinized, and rehydrated, followed by blocking of endogenous peroxidase with $3 \% \mathrm{H}_{2} \mathrm{O}_{2}$ (Sigma H-1009; SigmaAldrich Chemie GmbH, Steinheim, Germany) in PBS. Antigen was retrieved by heating the slides for $10 \mathrm{~min}$. Diluted biotinylated anti-BrdU antibody (BrdU In-Situ Detection Kit [\#550803]; BD Biosciences, San Diego, CA) or anti-Ki-67 antibody (Ki-67 clone-Tec 3 [\#M7249]; Dako, Glostrup, Denmark) were then applied and slides incubated for $1 \mathrm{hr}$ at $48^{\circ} \mathrm{C}$ in a humidified chamber. Then, ready-to-use streptavidin horseradish peroxidase complex (\#550803; BD Biosciences) was added, followed by a brief incubation with 3,3'-diaminobenzidine substrate (DAB, D-4293, Sigmafast; Sigma-Aldrich Chemie $\mathrm{GmbH}$ ). The tissue sections were counterstained in hematoxylin. Finally, BrdU and Ki-67-positive cells on representative slides were counted on four high-power fields for each animal.

For CD31 staining, 2- to 3- $\mu \mathrm{m}$ liver sections were dewaxed, rehydrated, and pretreated by boiling in $10 \mathrm{mM}$ citrate buffer, $\mathrm{pH} 6.0$, in a microwave oven. Endogenous peroxidase activity was blocked with $0.5 \% \mathrm{H}_{2} \mathrm{O}_{2}$ and $0.1 \% \mathrm{NaN}_{3}$. Sections were then (and following all subsequent steps) washed in Tris-buffered saline (TBS) and incubated for $60 \mathrm{~min}$ at room temperature with a rat-anti-mouse CD31 antibody (clone MEC 14.7; Abcam, Cambridge, UK), diluted 1:100 in TBS with $0.5 \%$ casein and 5\% normal goat serum. In negative controls, the primary antibody was replaced with antibody dilution buffer. A rabbit-anti-rat Ig secondary antibody (Dako) was then applied, followed by a polymer-based visualization system (Envision+; Dako), each for $30 \mathrm{~min}$. Finally, sections were developed in $0.02 \% 3,3^{\prime}-$ diaminobenzidine (Sigma, St. Louis, $\mathrm{MO}$ ) with $0.01 \% \mathrm{H}_{2} \mathrm{O}_{2}$, counterstained with hematoxylin, and mounted. Known positive controls were stained in parallel with each series.

To visualize liver steatosis, cryosections of $8-\mu \mathrm{m}$ thickness from liver tissue frozen immediately after removal at $-120^{\circ} \mathrm{C}$ were fixed in $4 \%$ paraformaldehyde. After dipping in $70 \%$ ethanol, sections were placed in Sudan Black solution for 20 min and rinsed afterwards in $70 \%$ ethanol and $\mathrm{H}_{2} \mathrm{O}$. Finally, sections were stained with nuclear fast red vector (H-3403; SigmaAldrich Chemie GmbH).

\section{Measurement of Liver Injury}

ALT and AST levels were measured by a photometric UV test measuring the oxidation of NADH to NAD (Roche Modular P800). Bile acids levels in the serum after $48 \mathrm{hr}$ post-PH were measured enzymatically using a Mira plus chemistry analyzer (Roche Diagnostics) with reagents from Trinity Biotech as previously described (34). Briefly, during oxidation of the bile acids to 3-oxo bile acids, equimolar quantity of NAD is reduced to NADH, which subsequently is oxidized to NAD. Nitroblue tetrazolium salt is then reduced to formazan, which has an absorbance maximum at $530 \mathrm{~nm}$. The concentration of bile acids in the sample is directly proportional to the intensity of the produced color.

\section{Hydroxyproline}

Quantification of hepatic hydroxyproline was measured as previously described (35). Briefly, after hydrolyzation of frozen liver tissue in $6 \mathrm{M} \mathrm{HCl}$ at $100^{\circ} \mathrm{C}$ for $16 \mathrm{hr}, 50 \mu \mathrm{L}$ was incubated with chloramine $\mathrm{T}(2.5 \mathrm{mM})$ for $5 \mathrm{~min}$ and Ehrlich reagent $(410 \mathrm{mM})$ for $30 \mathrm{~min}$ at $60^{\circ} \mathrm{C}$. Finally, absorption 
at $560 \mathrm{~nm}$ was measured and results expressed as micrograms per gram of wet liver tissue.

\section{Quantitative TaqMan PCR}

RNA was isolated from snap-frozen liver samples by Trizol Reagent according to the manufacturer's protocol (Life Technologies). cDNA was synthesized by using Omniscript RT kit 200 (cat. no. 205113; Qiagen) and mRNA analyzed by RT-qPCR (ABI 7900, SDS 2.3 software). Primers and probes sequences were ready-to-use kits from Applied Biosystems (Rotkreutz, Switzerland), reference gene control (RG) beta actin (\#Mm00607939_s1), IL-6 (\#Mm00446190_ml), TNF- $\alpha$ (\#Mm00443258_ml), GADD45 (\#Mm00432802_ml), and CDKN1a (=p21) (\#Mm00432448_m1). Relative changes in mRNA were calculated with the $\Delta \Delta \Delta \mathrm{Ct}$ method. Ct values of target gene expression (TG) was calculated relative to a RG using the following formula $\Delta \mathrm{Ct}_{\mathrm{TG}}=\mathrm{Ct}_{\mathrm{TG}}-\mathrm{Ct}_{\mathrm{RG}}$. Experimental groups (TG) were normalized to control group (CG): $\Delta \Delta \mathrm{Ct}=$ $\Delta \mathrm{Ct}_{\mathrm{TG}}-\Delta \mathrm{Ct}_{\mathrm{CG}}$, fold increase $=2^{-\Delta \Delta \mathrm{Ct}}$.

\section{Transmission Electron Microscopy}

To assess a putative effect of terlipressin treatment on hepatic sinusoids, respective cellular structures liver tissues were imaged by transmission electron microscopy. For that, liver samples were fixed in 5\% glutaraldehyde in PBS, postfixed in osmium tetroxide, stained en bloc in uranyl acetate, dehydrated, and embedded in epoxy resin. Ultrathin sections (50-100 nm) were analyzed with an EM12 transmission electron microscope (Philips, Eindhoven, Netherlands) equipped with a digital camera (Morada; SIS, Münster, Germany). Sinusoidal structures were analyzed blinded by an experienced liver pathologist (M.M.) for ultrastructural changes.

\section{Statistics}

All data are expressed as geometric means \pm standard deviations unless stated otherwise. For statistical analysis, Student $t$ test or two-way ANOVA test was used. Statistical analysis was performed with GraphPad Prism version 5.0 (GraphPad Software, Inc., La Jolla, CA). Data with $P$ less than 0.05 was considered as statistically significant.

\section{ACKNOWLEDGMENTS}

The authors would like to thank Anita Born and Cynthia Furer for technical support throughout the project.

\section{REFERENCES}

1. Jiang SM, Zhou GW, Zhang R, et al. Role of splanchnic hemodynamics in liver regeneration after living donor liver transplantation. Liver Transpl 2009; 15: 1043.

2. Marubashi S, Sakon M, Nagano H, et al. Effect of portal hemodynamics on liver regeneration studied in a novel portohepatic shunt rat model. Surgery 2004; 136: 1028.

3. Yagi S, Iida T, Taniguchi K, et al. Impact of portal venous pressure on regeneration and graft damage after living-donor liver transplantation. Liver Transpl 2005; 11: 68.

4. Michalopoulos GK. Liver regeneration after partial hepatectomy: critical analysis of mechanistic dilemmas. Am J Pathol 2010; 176: 2.

5. Doignon I, Julien B, Serriere-Lanneau V, et al. Immediate neuroendocrine signaling after partial hepatectomy through acute portal hyperpressure and cholestasis. J Hepatol 2011; 54: 481.

6. Abulkhir A, Limongelli P, Healey AJ, et al. Preoperative portal vein embolization for major liver resection: a meta-analysis. Ann Surg 2008; 247: 49.

7. Makuuchi M, Thai BL, Takayasu K, et al. Preoperative portal embolization to increase safety of major hepatectomy for hilar bile duct carcinoma: a preliminary report. Surgery 1990; 107: 521.

8. Ito K, Ozasa H, Noda Y, et al. Splenic artery ligation improves remnant liver function in partially hepatectomized rats with ischemia/ reperfusion injury. Liver Int 2007; 27: 400.

9. Umeda Y, Yagi T, Sadamori H, et al. Effects of prophylactic splenic artery modulation on portal overperfusion and liver regeneration in small-for-size graft. Transplantation 2008; 86: 673.
10. Ladurner R, Schenk M, Margreiter R, et al. Influence of portosystemic shunt on liver regeneration after hepatic resection in pigs. HPB Surg 2009; Article ID 83596.

11. de Franchis R. Revising consensus in portal hypertension: report of the Baveno $\mathrm{V}$ consensus workshop on methodology of diagnosis and therapy in portal hypertension. J Hepatol 2010; 53: 762.

12. EASL clinical practice guidelines on the management of ascites, spontaneous bacterial peritonitis, and hepatorenal syndrome in cirrhosis. J Hepatol 2010; 53: 397.

13. Treschan TA, Peters J. The vasopressin system: physiology and clinical strategies. Anesthesiology 2006; 105: 599; quiz 639-540.

14. Mukhtar A, Salah M, Aboulfetouh F, et al. The use of terlipressin during living donor liver transplantation: effects on systemic and splanchnic hemodynamics and renal function. Crit Care Med 2011; 39: 1329.

15. McCormack L, Petrowsky H, Jochum W, et al. Hepatic steatosis is a risk factor for postoperative complications after major hepatectomy: a matched case-control study. Ann Surg 2007; 245: 923.

16. Reddy SK, Marsh JW, Varley PR, et al. Underlying steatohepatitis, but not simple hepatic steatosis, increases morbidity after liver resection: a case-control study. Hepatology 2012; 56: 2221.

17. Kele PG, van der Jagt EJ, Gouw AS, et al. The impact of hepatic steatosis on liver regeneration after partial hepatectomy. Liver Int 2013; 33: 469.

18. Gabrielli M, Moisan F, Vidal M, et al. Steatotic livers. Can we use them in OLTX? Outcome data from a prospective baseline liver biopsy study. Ann Hepatol 2012; 11: 891.

19. Huang W, Ma K, Zhang J, et al. Nuclear receptor-dependent bile acid signaling is required for normal liver regeneration. Science 2006; 312: 233.

20. Asakura T, Ohkohchi N, Orii T, et al. Portal vein pressure is the key for successful liver transplantation of an extremely small graft in the pig model. Transpl Int 2003; 16: 376.

21. Li J, Liang L, Ma T, et al. Sinusoidal microcirculatory changes after small-for-size liver transplantation in rats. Transpl Int 2010; 23: 924.

22. Ding BS, Nolan DJ, Butler JM, et al. Inductive angiocrine signals from sinusoidal endothelium are required for liver regeneration. Nature 2010; 468: 310.

23. Asencio JM, Vaquero J, Olmedilla L, et al. "Small-for-flow" syndrome: shifting the "size" paradigm. Med Hypotheses 2013; 80: 573.

24. Moreau R, Lebrec D. The use of vasoconstrictors in patients with cirrhosis: type 1 HRS and beyond. Hepatology 2006; 43: 385.

25. Fausto N, Campbell JS, Riehle KJ. Liver regeneration. Hepatology 2006; 43: $S 45$.

26. Nicou A, Serriere V, Prigent $S$, et al. Hypothalamic vasopressin release and hepatocyte $\mathrm{Ca}^{2+}$ signaling during liver regeneration: an interplay stimulating liver growth and bile flow. FASEB J 2003; 17: 1901.

27. Vetelainen R, van Vliet AK, van Gulik TM. Severe steatosis increases hepatocellular injury and impairs liver regeneration in a rat model of partial hepatectomy. Ann Surg 2007; 245: 44.

28. Tian J, Huang H, Hoffman B, et al. Gadd $45 \beta$ is an inducible coactivator of transcription that facilitates rapid liver growth in mice. J Clin Invest 2013; 121: 4491

29. Buitrago-Molina LE, Marhenke S, Longerich T, et al. The degree of liver injury determines the role of p21 in liver regeneration and hepatocarcinogenesis. Hepatology 2013; doi:10.1002/hep.26412.

30. Lehmann K, Tschuor C, Rickenbacher A, et al. Liver failure after extended hepatectomy in mice is mediated by a p21-dependent barrier to liver regeneration. Gastroenterology 2012; 143: 1609.

31. May D, Djonov V, Zamir G, et al. A transgenic model for conditional induction and rescue of portal hypertension reveals a role of VEGF-mediated regulation of sinusoidal fenestrations. PLoS One 2011; 6: e21478.

32. Morsiani E, Aleotti A, Ricci D. Haemodynamic and ultrastructural observations on the rat liver after two-thirds partial hepatectomy. J Anat 1998; 192(Pt 4): 507.

33. Inderbitzin D, Studer P, Sidler D, et al. Regenerative capacity of individual liver lobes in the microsurgical mouse model. Microsurgery 2006; 26: 465.

34. Balmer ML, Joneli J, Schoepfer A, et al. Significance of serum adiponectin levels in patients with chronic liver disease. Clin Sci (Lond) 2010; 119: 431.

35. Patsenker E, Schneider V, Ledermann M, et al. Potent antifibrotic activity of mTOR inhibitors sirolimus and everolimus but not of cyclosporine A and tacrolimus in experimental liver fibrosis. J Hepatol 2011; 55: 388-398. 